\title{
What organisations around the world are doing to help improve doctors' wellbeing
}

The covid-19 pandemic has increased the pressure and stress that healthcare professionals are under. Now, more than ever, it is important that clinicians look after their wellbeing. Doctors from around the world explain what they have been doing to support their colleagues

Abi Rimmer, Cat Chatfield

The BMJ

\section{Keeping staff connected}

Lucy Warner, chief executive of NHS Practitioner Health, says, "At this difficult time for doctors and the NHS workforce it feels natural that we at Practitioner Health explore how we can use our experience to support and help.

"We're focusing initially on enabling staff to connect, as we feel many of the anxieties and fear being expressed will be similar for many people. With this in mind, we're creating a range of online events, webinars, and groups to act as a space to talk, share, learn, and care for one another.

"These groups include nightly mindfulness, a singalong, and yoga, as well as safe spaces we're calling 'common rooms' for staff groups to come together. These started on 6 April for doctors and will run twice daily, including weekends, at $11 \mathrm{am}$ and $6 \mathrm{pm}$ They'll be led by experienced Practitioner Health clinicians, and doctors can access them as often as they like. We hope to have other staff groups running soon.

"We've been overwhelmed by offers of support and so have built up a range of resources. This includes access to Silvercloud (cognitive behavioural therapy self-care modules) for all NHS staff and their families, as well as other apps such as Sleepio, Daylight, and Headspace.

"We've also identified a range of therapeutic networks run by accredited professionals who are offering free access to therapy and listening services for NHS staff and have included the links to these on our site.

"As part of the national offering being developed through NHS England we'll be exploring how we can tailor both individual and group interventions for those experiencing difficulties.

"We're aware that many won't need our help right now, but in the months to come may find the time is right to seek support, and so we're planning our programme of activity to continue into next year and beyond.

"The most important message we have for doctors is to take care of yourselves-you are human, you need to eat, rest, and recharge. If you need help now, we are here for you. If you need help later, we will be there for you too."

Access to resources and events here: www.practitionerhealth. nhs.uk/covid-19-workforce-wellbeing.

\section{Learning from the Italian experience}

Guido Veronese, associate professor of psychology at the department of human sciences and education, University of Milano-Bicocca, says, "The first lesson to be learnt from the early weeks of the crisis in Italy is that, in times of normality, far more resources should be allocated to promoting health personnel's mental health, wellbeing, and preparedness to efficiently respond to psychological emergencies.

"The magnitude of the stressors that health workers are currently being called to cope with clearly implies that psychological wellness is not an irrelevant or secondary concern. The more health workers are skilled at comprehending and managing their own psychological and emotional states, the more effectively they'll meet personal and collective challenges during and after catastrophic events. Consequently, worker burnout and loss of efficiency will be prevented.

"A second lesson is that national and regional health systems need to take responsibility for organising and coordinating the response to mental health providers' psychological needs during the crisis, providing a centralised public service in collaboration with local psychological services and ensuring 24 hour psychological support for helpers, as well as specialised services in the areas of stress and trauma management.

"As far as we are aware, such a crucial coordination effort is currently lacking in our own region of Lombardy, with the risk that fragmented and iatrogenic responses will be offered in place of effective interventions. A culture of psychological preparedness, clear guidelines following Inter-Agency Standing Committee recommendations, ${ }^{1}$ and coordinated intervention by regional and national authorities are urgently needed to protect health providers' mental health."

\section{Training in new skills}

Consultant anaesthetists, Ian Baxter, Rachael Bird, Nicola Hirschauer, and Karuna Kotur; and Cathy Lawson, fellow in environmentally sustainable anaesthesia; Roopa McCrossan, locum consultant anaesthetist; Suzy O'Neill, consultant in anaesthesia and intensive care; and Linda Waddilove, consultant anaesthetist, say, "In early March we formed the Freeman 
Hospital perioperative and critical care wellbeing group at Newcastle Hospitals NHS Foundation Trust. Our approach was structured around three pandemic phases: anticipatory, active, and post event. We have currently enacted the anticipatory stage. "Changing role expectations and correct personal protective equipment (PPE) use were found to be the main staff concern in the anticipatory phase and were our first priority.

"A 'round robin' training session was held to teach people skills that they might need during the outbreak but which were outside of their usual practice. These included things such as donning and doffing of PPE, intubating a patient, cardiac arrest in a patient with covid-19, transferring a patient with covid-19, and proning a covid-19 patient.

"We also offered tips on managing stress, sleep hygiene, and night shift working, as well as introducing acceptance and commitment therapy strategies to help manage anxiety.

"Around 500 staff have been trained to date. In parallel, we're working on practical support such as rest facilities, refuel and refresh boxes, and car parking provision.

"There is, however, only so much we can do for ourselves. Joining forces with our clinical psychology team has been fundamental. They've created training videos to help us to provide psychological first aid and have arranged for the provision of one-to-one support where necessary. While we hope that these measures will minimise the psychological impact of the pandemic, we recognise that, sadly, ongoing professional psychological assistance will likely be required in the aftermath.”

\section{Proper protective equipment}

Min Zhang, occupational health professor at the School of Public Health, Chinese Academy of Medical Science and Peking Union Medical College, China, says, "As a professional who experienced covid-19 several months ahead of the UK, in my point of view, the goal of mental health for staff could not be achieved without comprehensive measures.

"Appropriate PPE based on risk assessments is essential, especially since we are yet to know all about covid-19. All patients should be regarded as potentially having the virus.

"In China, all staff at healthcare facilities must wear medical surgical masks at the very least. Precautionary measures can make health workers feel safer.

"PPE is just one part of mental health protection though-holistic support systems should also be provided. Psychological support has been emphasised a lot, but it is much harder to do in practice. Simply communicating with and encouraging team members, possibly through peer support programmes or buddy systems, can help to overcome negative emotions.

"Support with living arrangements is also crucial. Chinese local authorities requisitioned hotels near hospitals to provide accommodation for staff, as well as healthy food, transportation to and from the hospital, and other daily necessities. Providing accommodation also meant that any staff who were infected could live away from their families and prevent the infection from spreading. Meanwhile, employers helped family members of health workers who were in need, as best as they could."

\section{Supportive communication}

Sukhpreet Singh Dubb, specialty trainee year 3 in oral and maxillofacial surgery at Addenbrooke's Hospital, says, "Our department created a dedicated WhatsApp group which had clear rules from the outset. This was not a social group but one dedicated to the wellbeing of those within the department. Daily updates were inevitably shared of more infections, more admissions, more complications, and, unfortunately, more deaths. Despite this, our group was also able to provide supportive messages, as well as up to date and accurate information. Anyone who became ill or had to self-isolate could update our department faster than any email or phone contact while other members could step in to fill the gap.

"It's in our nature to fall back on formal means of communication during a crisis. This is understandable since we use social media for very different reasons. Technology, however, gives us the power to take care of each other at a distance. Evidence shows that, when used appropriately, social media such as WhatsApp can reduce loneliness and improve psychological wellbeing. Both essential and important factors during this pandemic."

\section{Offering support online}

Antoni Calvo, psychologist and director of the Galatea Foundation, Barcelona, and Anna Mitjans, project manager for the foundation, say, "The Galatea Foundation-which provides assistance programmes for health professionals with mental illness or addictive behaviours-has directed its resources towards giving psychological support to health and social care professionals through an online platform, with the help of the General Board of Doctors, Psychologists, and Nurses; the Health and Consumption Ministry; and La Caixa Foundation.

"This videoconference service is provided by psychologists and psychotherapists who are carefully selected by the Galatea Foundation. It began in Catalonia but was expanded to the rest of Spain in April.”

"These interventions are delivered in a targeted and brief manner. They last between 20 and 30 minutes, unlike conventional psychological interventions. Psychiatric assessment is also provided, when needed, using pre-existing specialised mental health services.

"Interventions targeted at healthcare teams are also offered, focused on promoting their capability to cope with the situation and incorporating stress management tools to enhance their performance in this new reality.

"Healthcare professionals have also been provided with a constantly updated toolbox on the Galatea Foundation and Galatea Clinic websites, containing a wide range of resources to improve stress management and to lower anxiety and distress."

\section{Wellbeing suites}

Julie Gordon, emergency medicine consultant, NHS Ayrshire and Arran, says, "Our wellbeing initiatives are supported by medical peer support, clinical psychology services, staff support, and chaplaincy. We aim to provide a safe space for all staff to access, where they will find a variety of facilities to support their physical and emotional wellbeing.

"We have staff wellbeing suites in two of our hospitals that are open 24 hours a day, every day. These offer meals for staff who are unable to go home, a bedroom, and a restroom, as well as toiletries, games, and exercise equipment.

"We've also suggested that, as well as these staff wellbeing areas, each ward thinks about creating a safe space or a 'wobble zone.' This doesn't have to be a large space but somewhere staff can go to grab a drink, a bite to eat, or be alone if they want. 
We're all human and there will be times where we'll all just need a few moments to breathe."

\section{Wobble rooms}

Richard Duggins, consultant psychiatrist in medical psychotherapy and clinical lead (North East and Cumbria) NHS Practitioner Health, says, “'Wobble rooms' provide psychological first aid as staff rise to the challenges and adapt quickly. They create an emotional space to strengthen camaraderie and support. They care for those who care, and help to make the unbearable bearable.

"In our mental health and disability trust, wobble rooms are run both virtually and physically. The virtual rooms use Microsoft Teams to offer small facilitated 30 minute groups, three times a week.

"These online groups are led by two experienced facilitators and encourage attendees to discuss how things are for them now. Honest expression of feelings, and the exploration of overlaps between professional and personal life is encouraged. Every session ends with each attendee invited to identify one thing they are going to do that day to care for themselves.

"Wobble rooms also have a physical presence with our hospital sites having a designated social space for staff where they can go if they are having a 'bit of a wobble' or just need 10 minutes away. These welcoming and comfortable rooms are stocked with drinks, food, and nice things. A flipchart on the wall of each room encourages staff to share their thoughts and feelings, messages of support, or things to be grateful for. Drop-in, facilitated staff support groups are also delivered here.

"Initial feedback suggests our wobble rooms reduce feelings of isolation and confusion by providing a space to acknowledge the emotional impact of work, and they help staff not to neglect their physical and emotional care."

\section{Offering psychological support}

Mohammed Aldossary, specialist in paediatric dentistry at the Ministry of Health, Riyadh, Saudi Arabia, says, "The Saudi Commission for Health Specialties (SCFHS) is responsible for supervising and evaluating training programmes, as well as setting controls and standards for the practice of health professions.

"Currently, SCFHS is providing online lectures about covid-19, including one titled Managing the Psychological Crises and Anxiety Associated with the Corona Pandemic Among Health Practitioners.

“The SCFHS, through its 'supporter programme,' is also providing psychological support to all health practitioners throughout the kingdom. This initiative aims to alleviate the psychological pressures that healthcare workers face as our first line of defence in dealing with this pandemic.

"The Ministry of National Guard-Health Affairs (MNGHA) is also providing a staff wellbeing hotline. Given the impact of the pandemic on healthcare systems, the MNGHA leadership appreciates that it is essential to support all aspects of their employees' health, including mental health."

\section{Facilitated reflection}

Olivia Donnelly, consultant clinical psychologist at North Bristol NHS Trust, says, "At my trust we've been offering a range of support. This includes Me+MyTeam, a 40 minute psychology coaching session to support managers in supporting the psychological wellbeing of their colleagues. It combines advice on psychological safety, compassion focused therapy, peer to peer debriefing, and how to signpost for support to access specialist psychological interventions (including trauma focused support) as needed.

"We're also using the three step team procedure. This is a protocol developed by senior medics, the quality improvement team, and psychologists that enables staff to come together during the day and check in. It acts like an expanded version of a safety briefing, and there is the opportunity for peer to peer debriefing, as well as access to trauma screening.

"We've also instigated the \#20minCareSpace—a brief facilitated space to support staff to reflect on the impact of their work, discuss what's going well, and check in on their self-care.

We also offer our staff access to one-to-one psychology sessions. Our sense is that requests and need for this will come later during the trajectory once things start to ease and people feel more able to process their emotions."

\section{Supporting those with mental disorders}

Eugeni Bruguera, Galatea Clínic and Department of Psychiatry of Vall Hebron University Hospital; Gustavo Tolchinsky, Barcelona Medical Council-Association; and María Dolores Braquehais, Galatea Clinic, say, "Health professionals with mental disorders have an increased risk of suffering from distress under stressful circumstances. Experience from specialised treatment programmes ${ }^{2}$ may enrich the discussion on the psychological impact of the coronavirus pandemic among health professionals. ${ }^{3}$

"The Galatea Clinic in Barcelona has a great deal of experience in the treatment of mental disorders, including addictions, in health professionals. ${ }^{4}$

"Since the beginning of the pandemic, the clinic has tried to keep the balance between supporting health professionals in treatment while also responding to increasing new demands. At the same time, we're following preventive measures, including social distancing, as most health professionals are likely to be exposed to the virus.

"Remote interventions, by telephone or videoconference, have allowed our interdisciplinary team (psychiatry, psychology, nursing, and social work) to continue providing individual and group therapy.

"Cases in treatment on our high-risk-of-suicide and high-risk-for-practice programmes are now receiving extra support. Those under mandatory treatment are also being closely supervised in the workplace.

"A daily home treatment service, using videoconferencing and on site support (if needed), has been developed for acute psychopathological conditions that can still be managed at home. Inpatient admissions are only offered for severe cases that cannot be treated with this type of intervention.

"All healthcare professional programmes will need to adapt their interventions to the new challenges presented by this pandemic, in the short and long term."

\section{Control over working patterns}

Emma Challans, director of culture and improvement, and L Ruwani Abeyratne, RCP chief registrar, specialty registrar geriatric and general internal medicine, say, "Sherwood Forest Hospitals NHS Foundation Trust has developed a four level self-care and wellbeing strategy. Co-created with consultant psychologists and other key stakeholders, it's a strategy that 
recognises international evidence on mental health and psychosocial support ${ }^{5}$ in emergency settings and applies guidance from national psychology networks and institutes.

"Recognising what matters to doctors has been vital to offering robust and dynamic interventions, as explored in both the General Medical Council's Caring for Doctors, Caring for Patients $^{6}$ and the Royal College of Physicians' Keeping Medicine Brilliant.

"Allowing doctors to collaboratively rewrite their rotas and proactively coordinate staffing on a daily basis engenders a sense of control and autonomy in a rapidly changing and unfamiliar environment. Clear and transparent communication signposts doctors to up-to-date information, both clinical and non-clinical, reiterating the message that we are all in this together.

"Facilitating training using a multi-modal approach, in a range of skills, recognises that working within a sphere of competence is a fundamental professional value, especially at a time when many are required to work outside of their usual practice. Underpinning this is the importance of acknowledging the psychological distress that many doctors are exposed to in such difficult times. Hosting themed workshops, providing designated safe spaces, and ensuring that support systems are communicated to doctors breaks down pre-existing barriers and reiterates that the health and wellbeing of doctors is paramount in our response to covid-19."

1 Interim briefing note addressing mental health and psychosocial aspects of covid-19 outbreak (developed by the IASC's Reference Group on Mental Health and Psychosocial Support). https://interagencystandingcommittee.org/iasc-reference-group-mental-healthand-psychosocial-support-emergency-settings/interim-briefing.

2 Braquehais MD, Tresidder A, DuPont RL. Service provision to physicians with mental health and addiction problems. Curr Opin Psychiatry 2015;28:324-9. 10.1097/YCO.0000000000000166 26001924

3 Lai J, Ma S, Wang Y, etal . Factors associated with mental health outcomes among health care workers exposed to coronavirus disease 2019. JAMA Netw Open 2020;3:e203976. 10.1001/jamanetworkopen.2020.3976 32202646

4 Tolchinsky G, Padros J. Caring programmes for sick doctors are a crucial step to self-regulation. The BMJ opinion. 25 September 2019. https://blogs.bmj.com/bmj/2019/ 09/25/caring-programmes-for-sick-doctors-are-a-crucial-step-to-self-regulation.

5 https://interagencystandingcommittee.org/system/files/2020-03/MHPSS\%20COVID19\% 20Briefing\%20Note\%202\%20March\%202020-English.pdf

6 General Medical Council. www.gmc-uk.org/-/media/documents/caring-for-doctors-caringfor-patients_pdf-80706341.pdf

www.rcplondon.ac.uk/guidelines-policy/keeping-medicine-brilliant

Published by the BMJ Publishing Group Limited. For permission to use (where not already granted under a licence) please go to http://group.bmj.com/group/rights-licensing/ permissions 